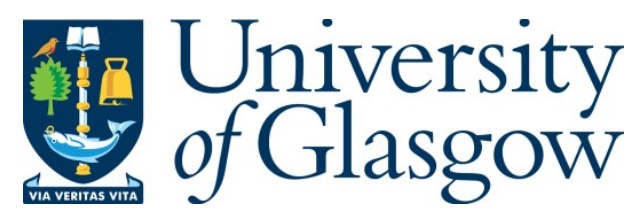

Woodgate, M. A., Shi, Y., Fitzgibbon, T., Barakos, G. N. and Li, P. (2021) Simulation of step input in collective pitch for hovering rotor. Aerospace Science and Technology, $110,106425$.

(doi: 10.1016/j.ast.2020.106425)

This is the Author Accepted Manuscript.

There may be differences between this version and the published version. You are advised to consult the publisher's version if you wish to cite from it.

https://eprints.gla.ac.uk/226967/

Deposited on: 7 December 2020

Enlighten - Research publications by members of the University of Glasgow http://eprints.gla.ac.uk 


\title{
Simulation of Step Input in Collective Pitch for Hovering Rotor
}

\author{
Mark A. Woodgate ${ }^{1}$, Yongjie Shi ${ }^{2}$, Thomas A. Fitzgibbon ${ }^{1}$, George N. \\ Barakos ${ }^{1}$, and Pan $\mathrm{Li}^{2}$ \\ ${ }^{1}$ CFD Laboratory, School of Engineering, University of Glasgow, \\ Glasgow, G12 8QQ, Scotland, UK \\ ${ }^{2}$ National Key Laboratory of Rotorcraft Aeromechanics, Nanjing \\ University of Aeronautics and Astronautics, Nanjing 210016, China
}

\begin{abstract}
Advanced CFD tools are nowadays used routinely for analysis and design of rotorcraft with the research community shifting towards simulations of rotorcraft during maneuvering flight. One of the impediments of this effort is the lack of data for validation, evaluation and thorough assessment of CFD methods when it comes to rotors with time-varying control inputs. This paper presents a first effort to validate CFD tools for step-inputs in rotor control angles against previously un-published experimental data. The experimental study was carried out at the Nanjing University of Aeronautics and Astronautics in China and consists of time varying control inputs for a hovering rotor. The agreement between the simulation and experimental study is good and quantify the overshoot in loads for this dynamic case. The results examine the lag in loads response to and the dynamic response of the wake with recommendations on spatial and temporal resolution to capture these effects.
\end{abstract}

\section{Nomenclature}

$\beta \quad$ Blade flap angle (rad) 
$\delta V \quad$ Boundary of the control volume $\left(\mathrm{m}^{2}\right)$

$\eta, \tau \quad$ Correction factors for non-uniform inflow

$\gamma \quad$ Lock number

$\Omega \quad$ Rotor rotational speed $(\mathrm{rad} / \mathrm{s})$

$\rho \quad$ Air density $\left(\mathrm{kgm}^{-3}\right)$

$\theta \quad$ Collective pitch $(\mathrm{rad})$

$\theta_{r} \quad$ Radial pitch angle (rad)

a Slope of lift coefficient vs angle of attack (per rad)

c $\quad$ Blade section chord (m)

$F_{i} \quad$ Inviscid flux

$F_{v} \quad$ Viscous flux

$l \quad$ Distance of blade center of mass from flapping hinge, $(\mathrm{m})$

$M_{\beta} \quad$ Total flap moment $(\mathrm{Nm})$

$m_{a} \quad$ Apparent additional mass of air influenced by rotor disk ( $\left.\mathrm{kg}\right)$

$m_{b} \quad$ Mass of rotor blades $(\mathrm{kg})$

$N_{b} \quad$ Number of rotor blades

$R \quad$ Rotor radius (m)

$S \quad$ Navier-Stokes equation source term

$T \quad$ Rotor thrust $(\mathrm{N})$

$t \quad$ Time $(\mathrm{s})$

$u, v, w$ Velocity components in Cartesian coordinates

$V \quad$ Control volume $\left(\mathrm{m}^{3}\right)$

$v_{\text {ind }} \quad$ Induced velocity $(\mathrm{m} / \mathrm{s})$

$W \quad$ Vector of conservative variables

ALE Arbitrary Lagrangian Eulerian

CFD Computational fluid dynamics

NUAA Nanjing University of Aeronautics and Astronautics 


\section{Introduction}

The rotor wake vortex is a dominant feature of the helicopter flow field and it has been identified as one of the primary aspects for predicting helicopter aerodynamics and flight performance [1]. The rotor wake exhibits an overall periodicity state, while it is highly unsteady and aperiodic during transient maneuvers. The unsteadiness arises from the time lag of the wake dynamic response induced by the ramp control inputs, and by unsteady fuselage motions fluctuations, etc. results in severe oscillatory airloads and helicopter response. The maneuvering capability is key for several helicopter operations and therefore, there have been a few efforts to develop methods and investigate these complex aerodynamic phenomena during maneuvering flight [2].

Over the past few decades, researchers have developed many analytical models for rotor or helicopter simulations for steady flight $[3,4,5,6,7,8,9,10,11]$, but most failed to simulate the inherent nonlinearities in transient maneuvers that are observed in dynamic response experiments $[12,13,14]$. To achieve this, a common approach is to account for the unsteady effects on the net thrust and moments. Carpenter [12] first developed a dynamic extension of momentum theory by introducing an apparent mass term in the thrust equation to model the inertia of the air. They investigated the effect of rapid increase in blade-pitch on rotor airloads and inflow. Following that research, several dynamic inflow models were developed and validated $[2,15,16]$. These models are widely used in flight simulation for their computational efficiency, but they provide no insight into the rotor wake physics. Therefore, these models are limited to applications where the rotor wake interaction is not severe, and only the rotor performance, namely thrust and power are concerned.

To explicitly take the wake geometry into account, one approach is via the timemarching wake method. In this method no assumptions regarding periodicity of the wake are made, that can deform under its own influence and external disturbances. Sadler [17] predicted the blade loads during steady turn flight by using a free-wake method with an explicit, Euler time-marching scheme. Bhagwat [18] [18] extended the Maryland free wake analysis and developed a time-accurate free vortex method by incorporating a second-order, predictor-corrector time-marching algorithm. They applied this methodology to investigate rotor wake response during several idealized maneuvering flight. The results showed that the overshoot in blade 
loads during transient maneuvering is caused by the dynamics of buildup in wake structure and induced velocity and is not by flow inertia [19]. In addition to the vortex filament wake models, Brown [20] and He [8] presented simulation results of rotor dynamic response to rapid change in collective pitch using vorticity transport method, and a viscous vortex particle model, respectively. These methods satisfactorily simulated the wake distortion and its effects, but the non-linearities around blade were not well captured, especially for modern rotors with complex geometry, due to the lift line or lift surface model are usually employed. Also, there are still some approximations and assumptions in predicting the creations, formations and roll-up of the wake vorticity in these methods.

The first principle based CFD methodology eliminates the requirements of modeling rotor wake and blade airloads. It has gained much attention in recent years, and there is an increasing application in design and analysis of rotorcraft with CFD methodology [21, 22, 23, 24], but there are very few works in maneuvering flight simulations. In [25, 26, 27, 28], researchers carried out the simulations of high-g pull-up maneuver, namely UTTAS 11029, from UH -60A flight test using coupling CFD/CSD method. Their results showed that the current method can capture the increase in the mean force during the manege, but the non-linearities in the normal force time histories were not satisfactorily predicted. The discrepancy may be attributed to the poor capture of dynamic stall events of retreating blade, as well the physics governing the stall. These researches also raised a question whether stateof-the-art CFD techniques can perform well in simulating the complex aerodynamic features of a rotor during aggressive maneuvering.

It is extremely difficult to simulate the dynamic response of complete helicopters during maneuvering flight apart from the capturing of the flow physics the elastic response of the blade plays a significant part in blade loading. When comparing the simulated results with the experimental data the discrepancy can from a wide range of possible causes, some of which may cancel each other out, making validating each part of the simulation difficult. The validation needs to be broken down into "easier" problems with a much reduced number of uncertainties, hence the need for simple dynamic test cases at realistic blade articulation rates of controlled manoeuvres.

For a first step on validating a CFD for maneuvering flight, we start from the dynamic response of an isolated rotor to rapid change in collective pitch which is the most fundamental cases include stop input in control. The other purpose of this 
paper is to exploit well-defined, idealized maneuvering flight experiment using the unique test rigid of Nanjing University of Aeronautics and Astronautics. The test provides reliable data for code validation.

\section{Numerical method}

\subsection{Dynamic inflow method}

To model the build-up of the inflow during rotor transient motions, a dynamic inflow equation is obtained by using combined momentum and blade element theory (annulus theory) [12]. Therefore,

$$
\begin{aligned}
T & =m_{a} \dot{v}_{\text {ind }}+2 \pi R^{2} \rho v_{\text {ind }}\left[v_{\text {ind }}+\frac{2}{3} \dot{\beta} R\right] \\
& =\frac{1}{6} N_{b} \rho \Omega^{2} c_{e} R^{2}\left[\theta_{r}-\frac{3}{2} \frac{c_{1}}{c_{e}} \eta \frac{v_{\text {ind }}}{\Omega R}-\frac{\beta}{\Omega}\right],
\end{aligned}
$$

where

$$
c_{e}=\frac{\int_{0}^{R} c r^{2} \mathrm{~d} r}{\int_{0}^{R} r^{2} \mathrm{~d} r}, \quad c_{1}=\frac{\int_{0}^{R} c r \mathrm{~d} r}{\int_{0}^{R} r \mathrm{~d} r}, \quad m_{a}=0.637 \rho\left[\frac{4}{3} \pi R^{3}\right] .
$$

$m_{a}$ is the apparent mass term with the addition of an impermeable circular disk accelerating in a stagnant fluid, and $\eta$ is a corrected factor for non-uniform inflow.

The blade flap dynamics equation is coupled to the dynamic inflow model to account for the effect of blade flap

$$
\ddot{\beta}+\Omega^{2} \beta=\frac{1}{2} \gamma \Omega^{2}\left[\frac{c_{2}}{c_{e}} \theta-\frac{4}{3} \tau \frac{v_{\text {ind }}}{\Omega R}-\frac{c_{2}}{c_{e}} \frac{\beta}{\Omega}\right] \equiv M_{\beta},
$$

where

$$
c_{2}=\frac{\int_{0}^{R} c r^{3} \mathrm{~d} r}{\int_{0}^{R} r^{3} \mathrm{~d} r}
$$

and $\tau$ is also a correction for non-uniform inflow. Equation 3 can be used to model a teetering hinge with the assumption that the blade is rigid.

To reduce the equation to first order, equation 3 is rewritten in a matrix form with two variables $\beta$ and $\dot{\beta}$,

$$
\frac{\mathrm{d}}{\mathrm{d} t}\left[\begin{array}{c}
\beta \\
\dot{\beta}
\end{array}\right]=\left[\begin{array}{cc}
0 & 1 \\
-\Omega^{2} & 0
\end{array}\right]\left[\begin{array}{l}
\beta \\
\dot{\beta}
\end{array}\right]+\left[\begin{array}{c}
0 \\
M_{\beta}
\end{array}\right] .
$$


The systems of differential equations 1 and 5 , can be solved by numerical methods, if values of $\theta$ and $\Omega$ are given against time. Then, the thrust can be computed from the time histories of the variables $v_{\text {ind }}, \beta$ and their derivatives,

$$
T=m_{a} \dot{v}_{\text {ind }}+2 \pi R^{2} \rho v_{\text {ind }}\left[v_{\text {ind }}+\frac{2}{3} \dot{\beta} R\right]-m_{b} l \ddot{\beta},
$$

where $m_{b}$ is the mass of the blades and $l$ is the distance of the blade center of mass from the flapping hinge.

Appendix A of the paper presents a MATLAB implementation of the simple dynamic model with the deployed parameters.

\subsection{Time-accurate free wake method}

The time-accurate free wake method developed in Reference [29] is utilized here to perform simulations complementary to CFD and to the model presented in the previous paragraph. A brief description of the time-accurate free wake method is given here for completeness. In this method, the wake vortices are force-free and convect with the local velocity, the motion of each vortex segment is governed by the convection equation

$$
\frac{\mathrm{d} r_{v}}{\mathrm{~d} t}=V_{v}\left(r_{v}\right)
$$

where $V_{v}$, is the local fluid velocity, it is composed of free-stream velocity and velocity induced by wake system and rotor maneuvers. A partial differential form of equation 7 is usually employed in numerical solutions, and is written as:

$$
\frac{\partial r_{v}(\psi, \zeta)}{\partial \psi}+\frac{\partial r_{v}(\psi, \zeta)}{\partial \zeta}=\frac{1}{\Omega} V_{v}\left(r_{v}(\psi, \zeta), t\right)
$$

The growth in vortex core radius is then approximated using

$$
\frac{\mathrm{d} r_{\zeta}}{\mathrm{d} t}=4 \alpha \nu \delta
$$

The blade is divided into several segments along its radius and each segment is modeled using the Weissinger-L lifting-surface model[29] Figure 1. The bound vortex is fixed at the 1/4-chord line and control points are located at the $3 / 4$-chord stations. The trailed vortex is assumed to be completely rolled up behind the blade, and the trailed vorticity is concentrated at the tip vortex which comprises the far-wake. The vortex strength in the near wake is computed from the no-penetration bound- 
ary condition at each control point. A Betz-type vortex sheet roll-up model is used to determine the initial conditions for equations 8 and 9 i.e., initial release point and core radius of the tip vortex. A second-order center difference and backward difference schemes with explicit artificial dissipative terms, named as CB2D scheme is adopted to perform the time marching solution. The blade flapping equation 5 is solved using an explicit time integration scheme. At each time step, these three equations are solved in sequence. Additionally, the time-accurate solution starts from the periodic steady-state solution from the relaxation technique to avoid numerical instability.

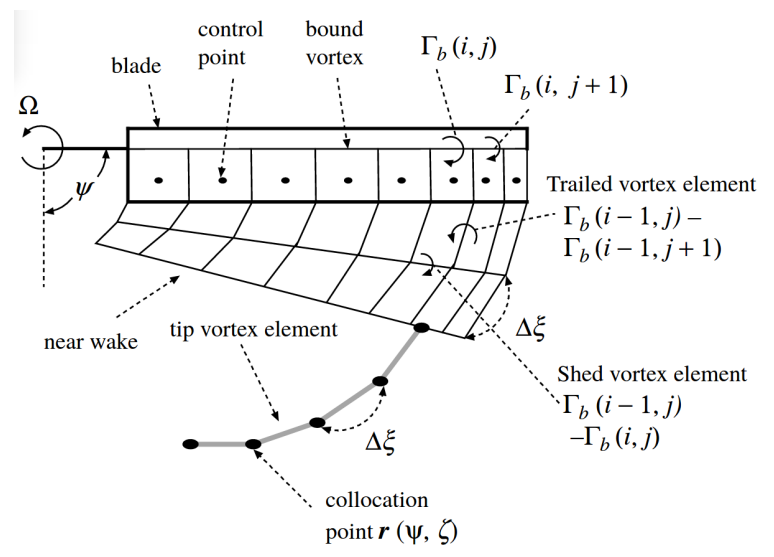

Figure 1: Sketch of the blade model, near wake and tip vortex[29].

\subsection{Computational Fluid Dynamics method (HMB solver)}

All calculations were performed using the parallel CFD solver HMB3 (Helicopter Multi Block) [30]. HMB3 solves the dimensionless 3D Navier-Stokes equations in integral form using the Arbitrary Lagrangian Eulerian (ALE) formulation for timedependent domains with moving boundaries:

$$
S=\frac{\mathrm{d}}{\mathrm{d} t} \int_{V(t)} W d V+\int_{\partial V(t)}\left[F_{i}(W)-F_{v}(W)\right] \cdot n d S
$$

where $V$ is the time dependent control volume, $\partial V$ is the boundary of the control volume, $W$ is the vector of conservative variables $(\rho, \rho u, \rho v, \rho w, \rho E), n$ is the unit vector on the boundary, $F_{i}$ and $F_{v}$ are the inviscid and viscous fluxes respectively.

The Navier-Stokes equations are discretized on the multi-block grid, using a cell-centered finite volume approach. A curvilinear coordinate system is adopted to simplify the formulation of the discretized terms, since body-conforming grids are 
adopted. The system of equations to be solved is:

$$
\frac{\mathrm{d}}{\mathrm{d} t}[W V]+R(W)=0 .
$$

In the above $W$ is the vector of conserved variables, $V$ denotes the volume of the cell and $R(W)$ represents the flux residual. Using a fully implicit time discretisation and approximating the time derivative by a second order backward difference equation (11) becomes

$$
\frac{3 V^{n+1} W^{n+1}-4 V^{n} W^{n}+V^{n-1} W^{n-1}}{2 \Delta t}+R\left(W^{n+1}\right)=0 .
$$

This equation is defined to be the unsteady residual $R^{\star}$. Following the original implicit dual-time approach introduced by Jameson [31] equation (12) is solved by iteration in pseudo-time $t^{\star}$. Using an implicit time discretisation on the pseudo-time $t^{\star}$ we can write

$$
\frac{W^{m+1}-W^{m}}{\Delta t^{\star}}=-\frac{R^{\star}\left(W^{m+1}\right)}{V}
$$

where the superscript $m+1$ denotes the time level $(m+1) \Delta t^{\star}$ in pseudo-time. The unsteady flux residual $\mathbf{R}^{\star}\left(W^{m+1}\right)$ is linearised in the pseudo-time variable $t^{\star}$ to form the sub iteration for solving equation 12 at each real $t$ time step.

Osher's upwind scheme [32] is used to resolve the convective fluxes for its robustness, accuracy and stability properties. The Monotone Upstream-centered Schemes for Conservation Laws (MUSCL) variable extrapolation method is employed in conjunction to formally provide second-order accuracy. The van Albada limiter [33] is also applied to remove any spurious oscillations across shock waves. The linear system is solved using a Krylov subspace algorithm, the generalized conjugate gradient method, with a block incomplete lower-upper (BILU) factorization as a preconditioner. The viscous stress tensor is approximated in HMB3 using the Boussinesq hypothesis. The two-equation turbulence model of $\mathrm{k}-\omega$ has been implemented into flow solver [34]. 


\section{Results and discussion}

\subsection{Rotor geometry and mesh}

Two model rotors are analyzed in this paper. One is a two-bladed teetering rotor tested in Nanjing University of Aeronautics and Astronautics (NUAA). The rotor has a rectangular and untwisted planform. The blade has a radius of $0.54 \mathrm{~m}$, aspect ratio $(\mathrm{R} / \mathrm{c}$ ) of 10 and solidity of 0.0637 . The NACA23012 airfoil is used throughout the blade. The other one is the model rotor tested by Carpenter in 1953 at NACA [12]. It is a conventional three-blade rotor with flapping hinges located at the center of rotation and drag hinges offset about $0.2286 \mathrm{~m}$ (9 in) from the center of rotation. The blade radius is $5.8 \mathrm{~m}(19 \mathrm{ft})$. The blades were made of plywood, were untwisted and had a NACA 23015 airfoil section. The rotor solidity was 0.042 . In the literature, only the planform view of rotor blades is given, and the blade used in simulation is based on another related paper [35].

For CFD analysis a C-topology mesh around the leading edge of the blade was selected, whereas an H-topology was employed at the trailing edge. For hover computations, only a single-blade domain was meshed, assuming periodic conditions for the flow field in the azimuthal direction. This assumption is valid if the wake generated by the rotor is assumed periodic and steady. Table 1 lists the grids employed for this study, showing the main meshing parameters and point distributions over the surface blade. The first cell normal to the blade was set to $8.0 \times 10^{-6} \mathrm{c}$ and $1.0 \times 10^{-5} c$ for the NUAA rotor and NACA rotor, respectively, which assures $y^{+}$less than 1.0 all over the blade for the employed Re. A blunt trailing edge was modeled using 42 mesh points. To capture the convection and distortion of tip vortex, the background mesh is refined with the minimize size of $0.03 \mathrm{c}$ in the wake development zone. A view of the computational domain along with the employed boundary conditions for hover is given in Figure 2.

\subsection{Test cases and simulation results}

\subsection{NACA tests}

To date, the only available data for validation of methods for cases with ramping rotor collective correspond to the tests by NACA [12]. Comparisons are show here with our simple dynamic inflow model and these are presented in Figures 3,4,5. 


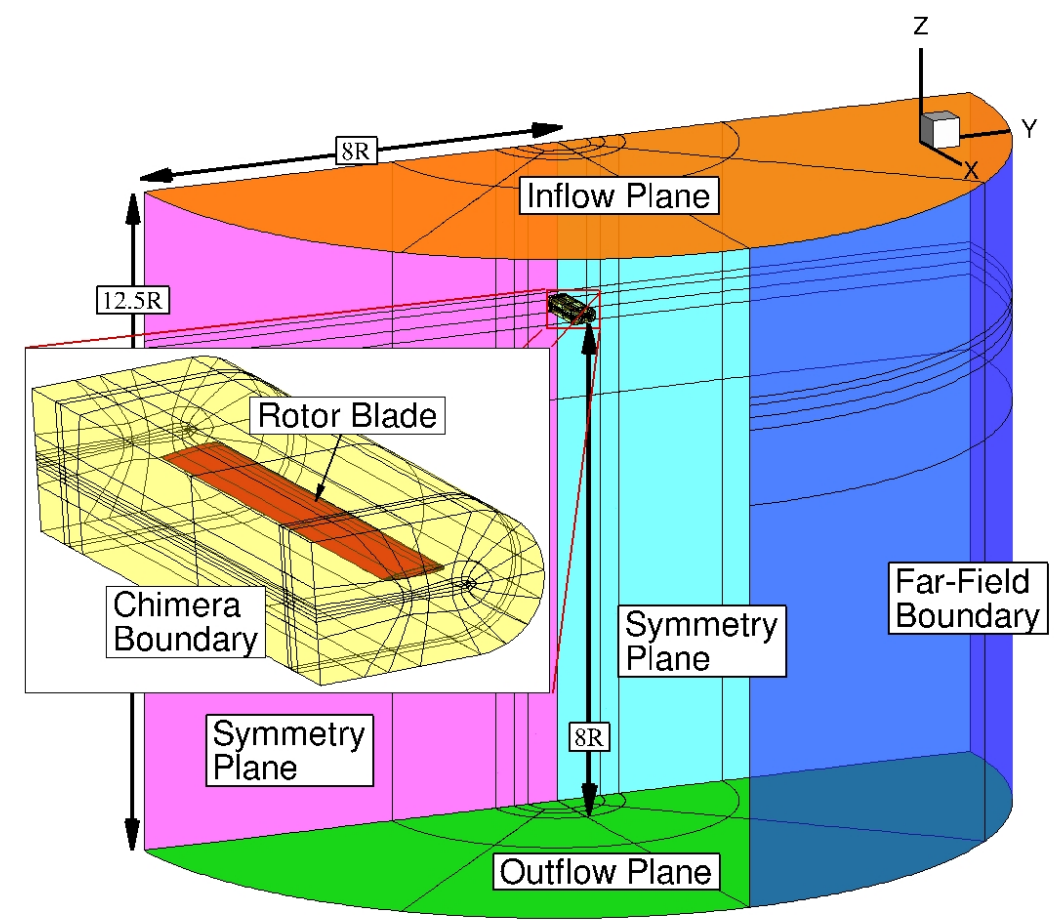

Figure 2: The computational domain, boundary conditions and multi-block topology of the NUAA rotor.

\begin{tabular}{cc}
\hline \hline Mode & NUAA rotor \\
\hline Background mesh size (cells) & 19.6 Million \\
Blade mesh size (cells) & 3.1 Million \\
Overall mesh size (cells) & 22.7 Million \\
Points along the span & 171 \\
Points around the airfoil & 250 \\
Steps per rotor revolution & 720 \\
Unsteady residual reduction & 3 Orders \\
\hline \hline
\end{tabular}

Table 1: Meshing parameters and unsteady parameters for the computational fluid dynamic calculation

This formulation correlates well with static experimental data but perform less well once dynamics are included. Some of this deficiency was overcome by also including the coupling between the collective and cyclic modes [36] which resulted in the Pitt-Peters model [37].

One of the key difficulties with the NACA test data is that the blade shape and especially its tip is not fully defined. This makes the case less than ideal for use with CFD tools where approximating the tip design, and hence the roll-up and trajectory of the tip vortex, adds extra uncertainty when comparing with the experimental results. Hence only a comparison with the simple dynamic inflow 
model is shown to demonstrate the effectiveness of the model. The results show that peak of the loads, the time of the thrust peak and the time of the maximum flap angle are not well predicted. In addition, the flap angle values after the peak are also poorly predicted. An important conclusion from the NACA tests is that the rapid ramping case of $200 \mathrm{deg} / \mathrm{sec}$ shows worst agreement with the model.

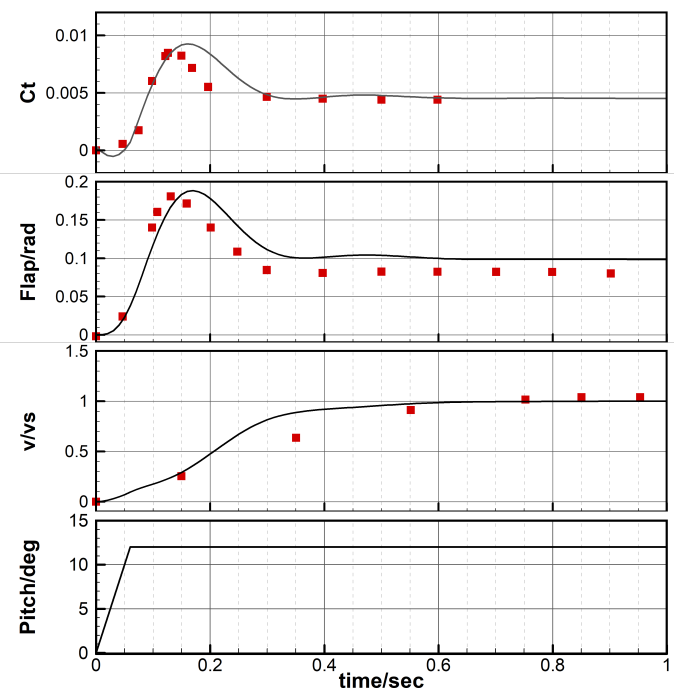

Figure 3: Change of collective pitch at a rate of 200 degrees/sec.

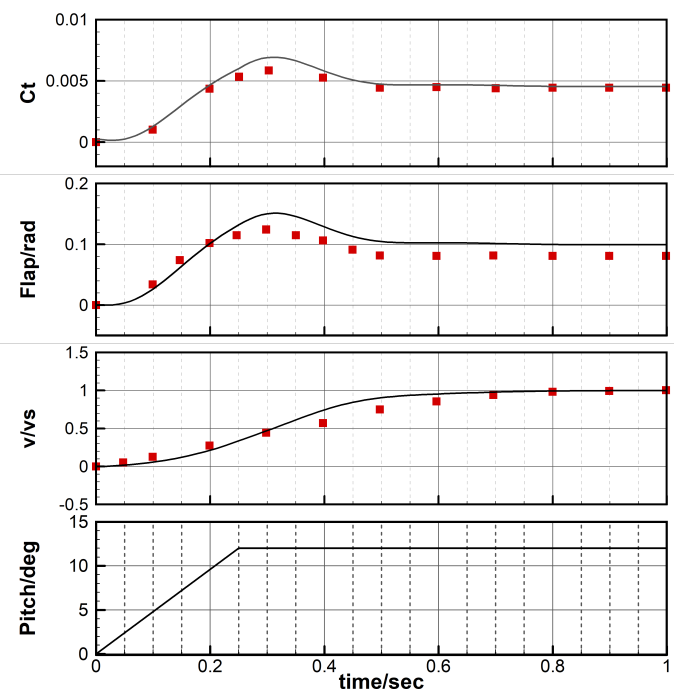

Figure 4: Change of collective pitch at a rate of 48 degrees/sec.

\subsection{NUAA tests}

The experiment of the rotor response to rapid change in control input was carried out using the whirling-beam rotor maneuvering flight test rig of the Nanjing University of Aeronautics and Astronautics. The test rig shown in 6 composes of a rotor 


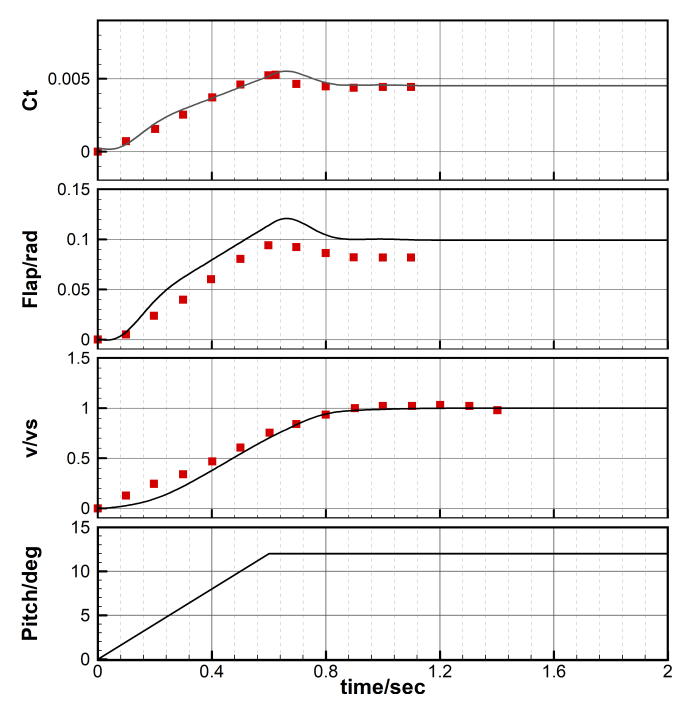

Figure 5: Change of collective pitch at a rate of 20 degrees/sec.

system, whirling beam and a pillar. The whirling beam is driven by a servo motor to rotate around the pillar axis. The model rotor was mounted at the end of the whirling beam and could perform rolling and pitching motions through adjusting the horizontal and vertical axis stepper motors. It could also rotate with beam to simulate the forward flight of helicopter. This unique set of experiments by NUAA has a wide range of conditions. The test is conducted using a well-defined blade geometry and the result is published in the Ph.D. thesis by Pan [35] in Chinese language. For this reason, a description of the test conditions and data obtained is given here.

In this experiment, a series of maneuvering flight tests including the rotor responses to rapid changes in control inputs (collective and cyclic pitches) and angular rate of pitching in hover and forward conditions were carried out. The result of step input in collective pitch is used in this paper. As listed in Table 2, the rotor operates at a rational speed of $1200 \mathrm{rpm}$. The change rate in collective pitch is $40 \mathrm{deg} / \mathrm{s}$. The pitch input begins at $0.1 \mathrm{~s}$ and last for $0.1 \mathrm{~s}$ too. Three collective settings of 0,2 , and 4 degrees were used.

\begin{tabular}{cc}
\hline \hline Parameter & Value \\
\hline Rotational speed (RPM) & 1200 \\
Starting time (sec) & 0.1 \\
Duration (sec) & 0.1 \\
Initial collective pitch (degrees) & $0,2,4$ \\
Rate of change of collective pitch (deg/sec) & 40 \\
\hline \hline
\end{tabular}

Table 2: Test conditions for the NUAA experiments 


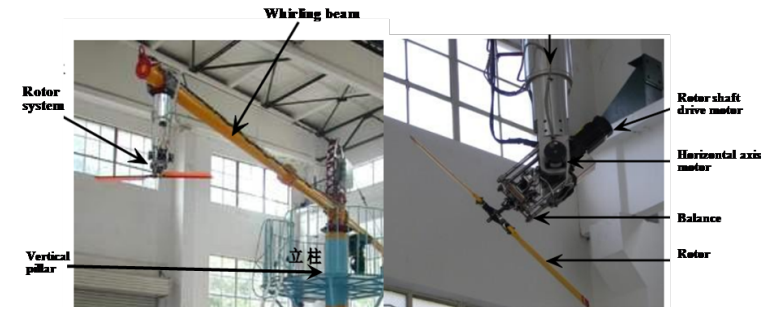

Figure 6: The whirling-beam rotor maneuvering flight test rig

The dynamic inflow model used in the previously was also exercised for this case. Overall, the results are in better agreement with the test data and suggest that the NUAA experiments, are somehow easier to reproduce with simulation methods. For all selected values of the initial collective, the CT peak, and the steady-state value after the ramping are well predicted. There is a difference in the times when the collective and thrust reach maximum, and this suggests that the wake of the rotor may be responding with a delay to the change of the collective.

Figures 7, 8 and 9 plot the experimental and calculation results of the rotor thrust response for three cases. The rapid increase of collectives started at 0.1 seconds and ended at 0.2 seconds. As shown in the figure, the rotor thrust increased linearly during collective input, and reached its maximum value at 0.2 seconds. After that, the thrust began to fluctuate and gradually reached a stable state within 0.5 seconds. The comparisons of three cases shown that the oscillation in thrust is smaller when the initial collective pitch increases. The time-accurate, free-vortex wake model simulated the overshoot and oscillation of the thrust, and predictions correlated well with experimental data.

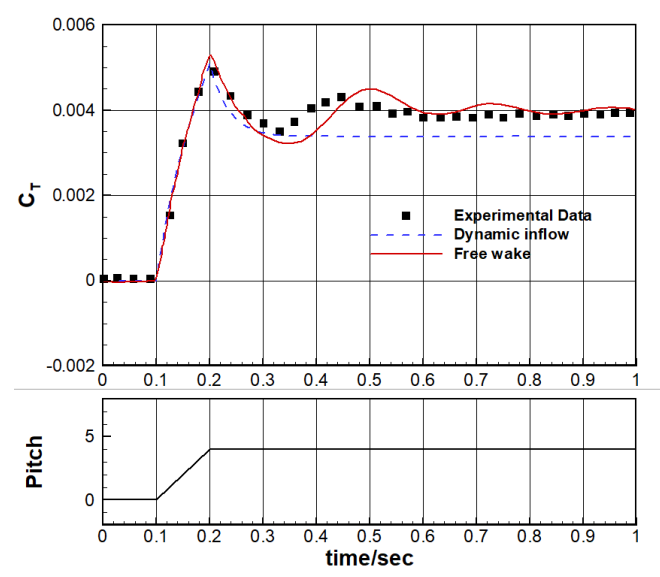

Figure 7: Comparison of the Thrust of the rotor for the NUAA test with initial collective of 0 degrees.

For this teetering rotor, the blades were rigid flap and the amplitude of elastic 


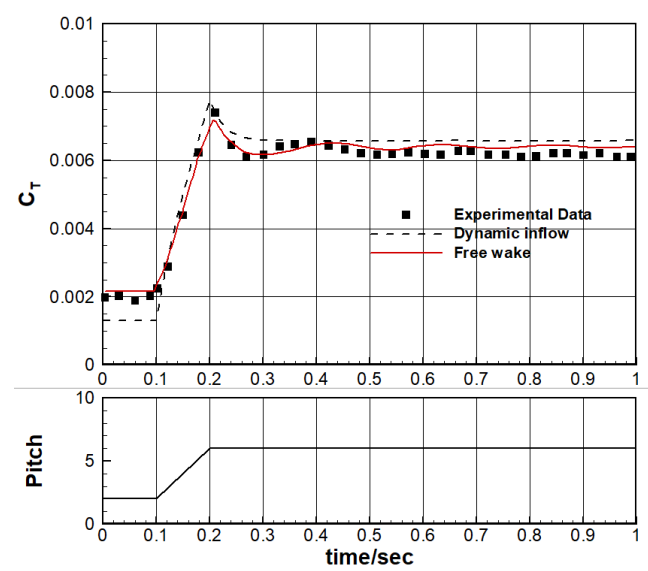

Figure 8: Comparison of the Thrust of the rotor for the NUAA test with initial collective of 2 degrees.

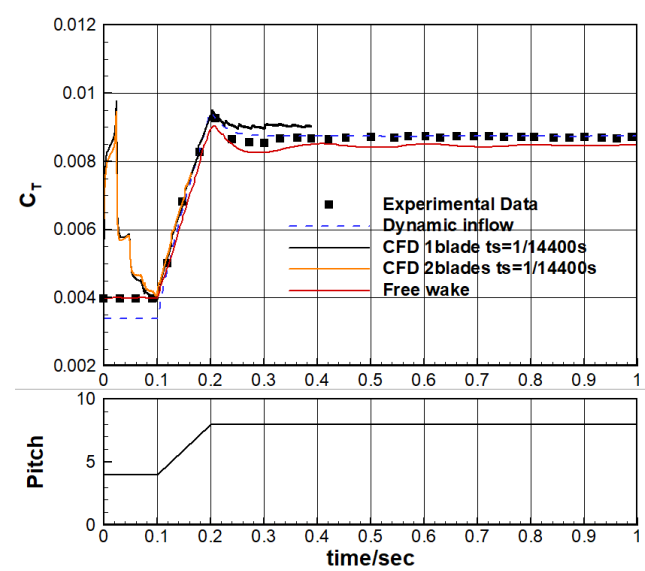

Figure 9: Comparison of the Thrust of the rotor for the NUAA test with initial collective of 4 degrees.

deformation flap is small and at high frequency, which had little impact on the timeaveraged rotor thrust. Therefore, the overshoot of thrust is mainly caused by a lag in the induced velocity. In dynamic inflow theory, the time lag of induced velocity is modeled by the additional mass of air. From the perspective of vortex theory, it is explained as the generation of wake vorticity and the transport lag behind the changes of collective and thrust.

Figures 10, 11, and 12 show the dynamic response of the rotor wake at three initial collectives. Taking the case of 0 degrees as an example, the influence of the wake distortion on rotor thrust is analyzed. The thrust is about zero initially and no wake is generated. Between 0.1s and 0.2s the rotor turns twice, and two revolutions of wake are generated and enter the flow field, so the induced velocity on the rotor plane is small. After 0.2 seconds, the collective input ends, and the ever growing wake enters the flow field and intertwines with the preceding wake, forming a vortex 

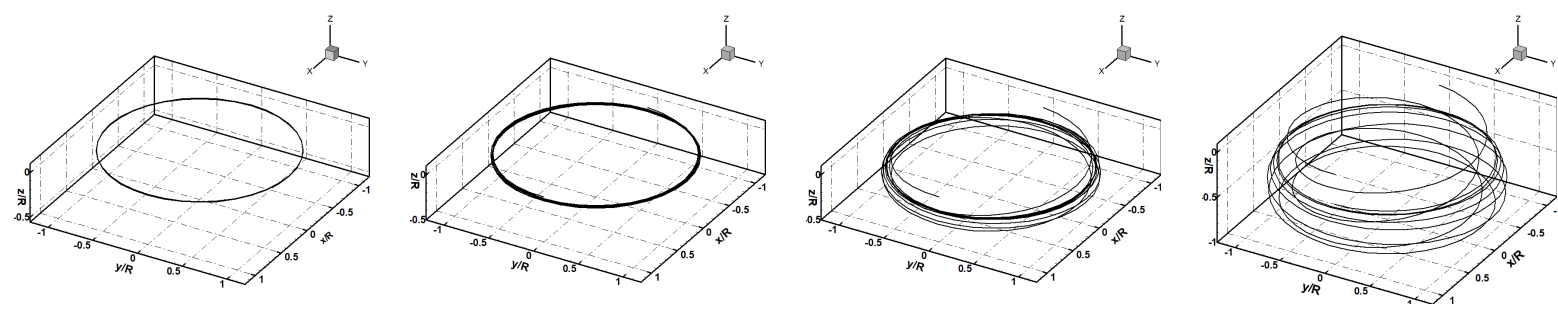

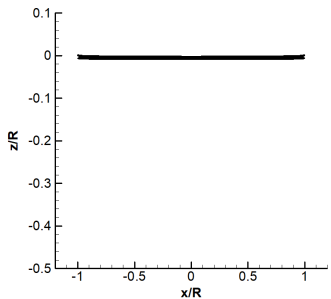

(a) $0.15 \mathrm{~s}$

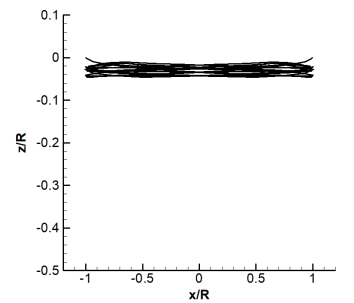

(b) $0.2 \mathrm{~s}$

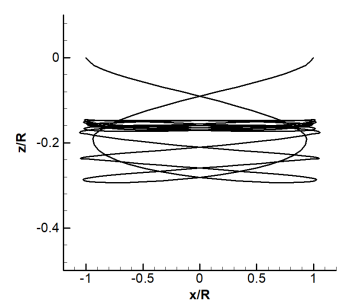

(c) $0.3 \mathrm{~s}$

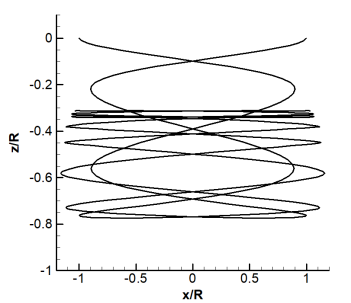

(d) $0.4 \mathrm{~s}$

Figure 10: The wake geometry from free-wake simulation with initial collective of 0 degrees.

ring with concentrated vorticity moving downstream. The continuous accumulation of vorticity increases the induced velocity at the rotor plane, resulting in a decrease of thrust. With the combined effect of local and self induced velocity, the concentrated vortex ring will form a type of spring like motion, which causes the fluctuation of induced velocity and thrust.

Comparing the geometry of the three wakes at same moment, shows that the velocity of the new wake vortex leaving the rotor plane and the concentration of vortices relates to the amplitude of collective pitch. The larger initial collective leads to larger velocity and lower vorticity concentration, and makes the oscillation amplitude of the rotor thrust smaller.

\subsection{CFD Simulations}

The CFD simulations employed the HMB3 tool of Glasgow University and focused on the NUAA experiments. Two cases are considered starting the ramp-up of the rotor from 2 and 4 degrees of collective. The experiments provided detailed time-traces of the collective versus time and the results were approximated using curve-fitting.

The simulations started with the 4 degree collective case so that the wake is produced by the rotor before the ramping is rather strong, coherent and relatively far from the blade. In addition, the computational domain included only one of the two blades of the rotor. This was mainly done for economy with the computations though a full rotor case with both blades present was also computed. The history of 

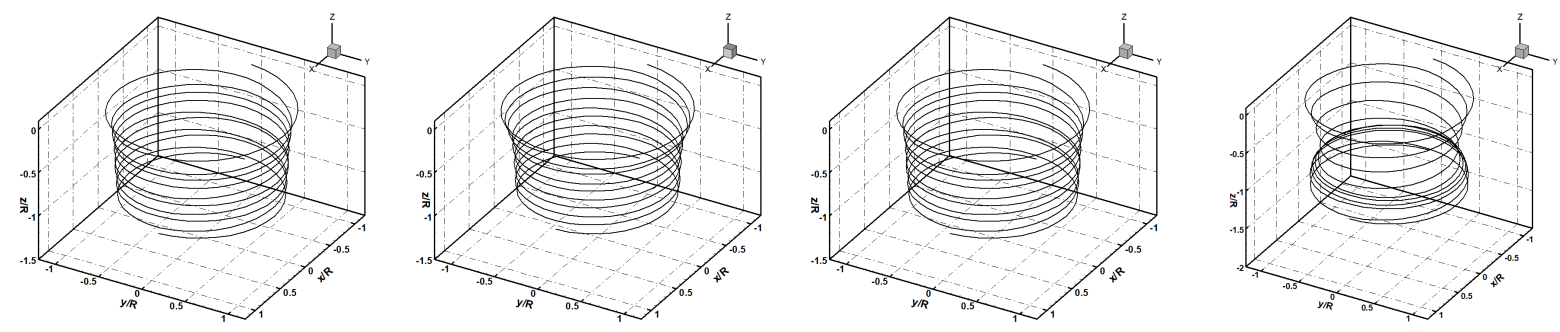

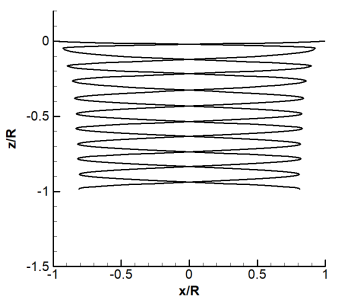

(a) $0.15 \mathrm{~s}$

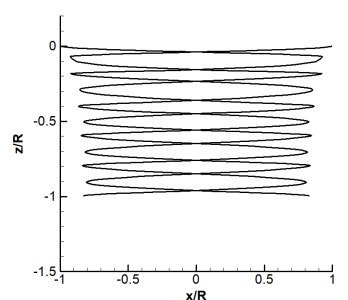

(b) $0.2 \mathrm{~s}$

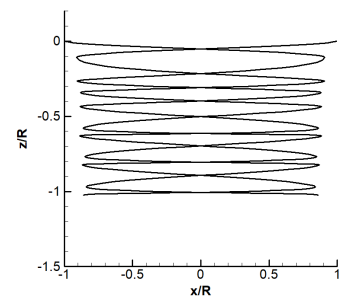

(c) $0.3 \mathrm{~s}$

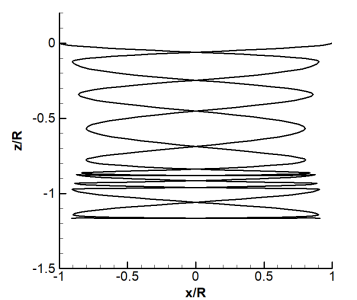

(d) $0.4 \mathrm{~s}$

Figure 11: The wake geometry from free-wake simulation with initial collective of 2 degrees.
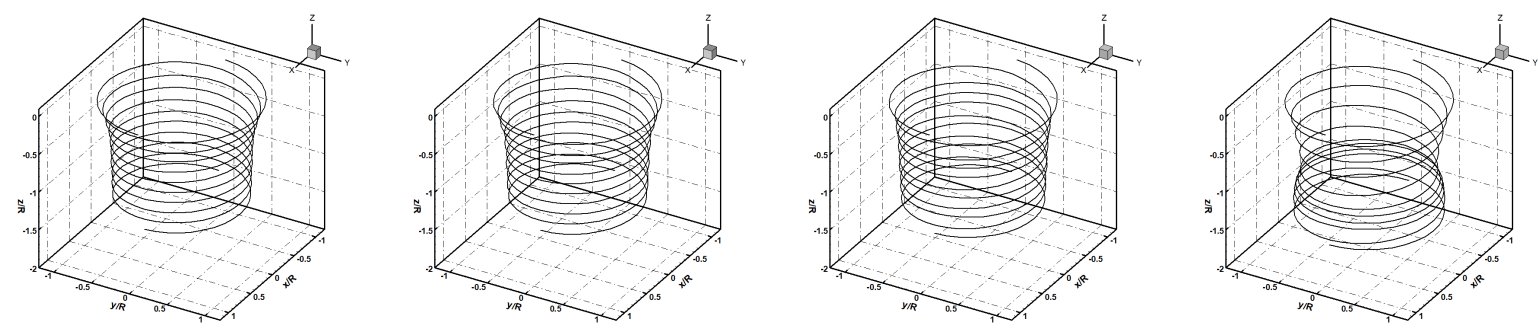

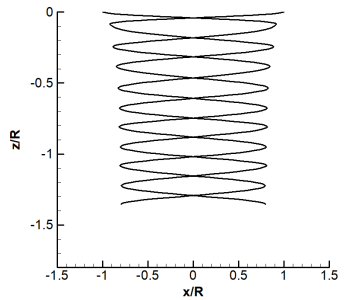

(a) $0.15 \mathrm{~s}$

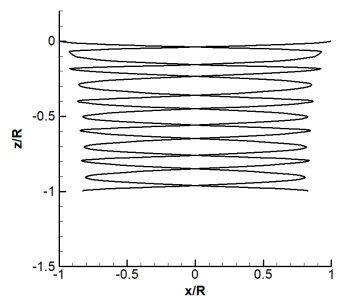

(b) $0.2 \mathrm{~s}$

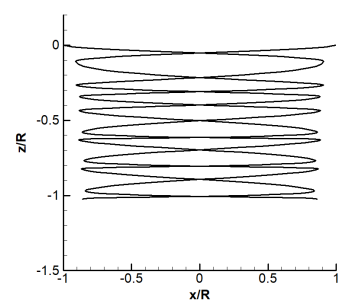

(c) $0.3 \mathrm{~s}$

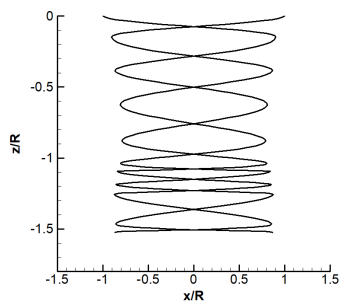

(d) $0.4 \mathrm{~s}$

Figure 12: The wake geometry from free-wake simulation with initial collective of 4 degrees. 
the collective and thrust can be seen in Figure 13 where the flow is seen to converge to the experimental thrust value just before the 0.1 seconds mark. The ramping is relatively fast, and the sampling of the experimental signal is rather coarse between 0.1 and 0.2 seconds. The results of the CFD method appear to track the change of the loads very closely and provide more details in the obtained signal. It is interest to note that the peak thrust value appears ever so slightly later than the peak of the collective. After the peak there is a period of about $0.05 \mathrm{~s}$ where the thrust drops before it begins to oscillate with a decreasing amplitude. Steady state is eventually reached after about $0.4 \mathrm{~s}$.

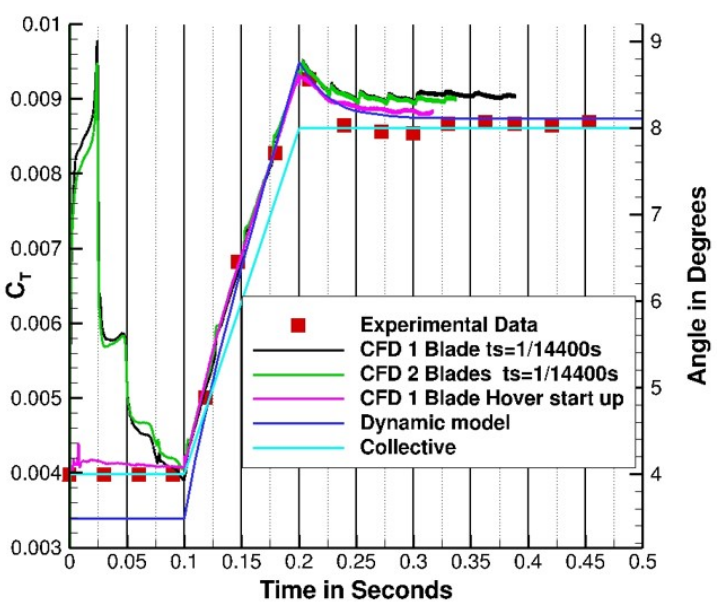

Figure 13: Comparison of the thrust of the rotor for the NUAA test with initial collective of 4 degrees for different CFD configurations.

The results for the complete rotor are cross plotted with the results for the single blade also shown figure 13 and no significant differences are observed demonstrating the validity of using single-bladed, periodic domain simulations. Both the complete rotor and single bladed simulations had slight blips in the thrust every $180^{\circ}$ when a free-stream initial condition was used. This shows that although using two rotor revolutions converges the thrust to the correct starting value its not enough to remove its transients. It was found that even using 6 and 8 revolutions before the collective pitch-up did not greatly improve the results, so another initialization method was used. This method used a fully converged steady state hover calculation as the initial conditions. This removed the small oscillations in the thrust and the recovery of the thrust after the blade has reached $8^{\circ}$ of collective more closely followed the experiment.

Figure 14 shows visualization of the rotor wake as a function of time. The 


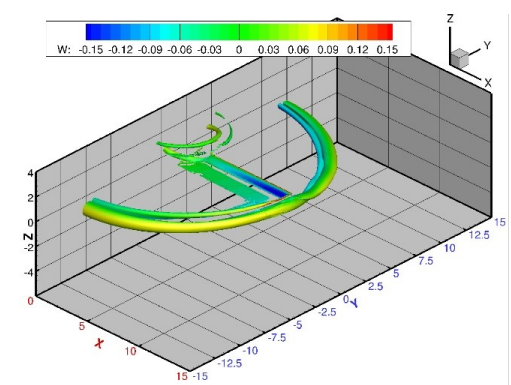

(a) $0.10 \mathrm{~s}$ (collective $4.00^{\circ}$ )

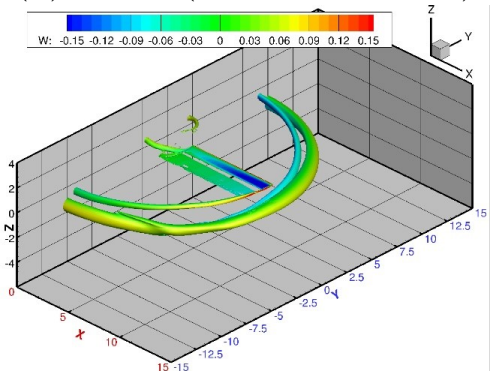

(d) $0.133 \mathrm{~s}$ (collective $5.33^{\circ}$ )

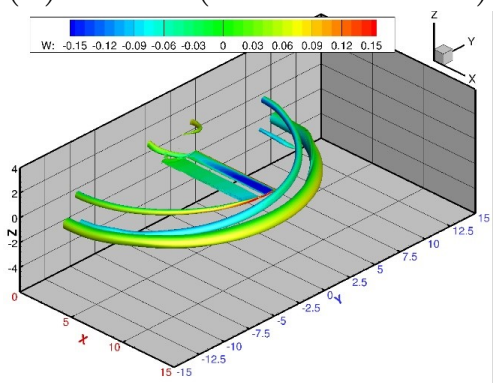

(g) $0.167 \mathrm{~s}\left(\right.$ collective $\left.6.67^{\circ}\right)$

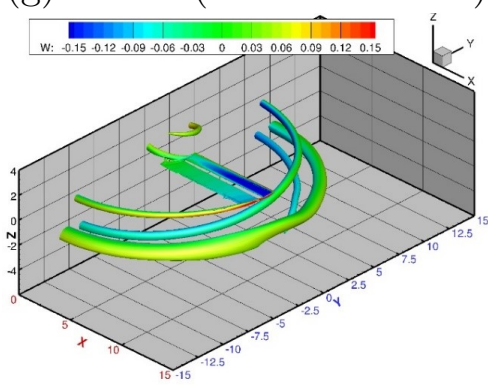

(j) $0.20 \mathrm{~s}$ (collective $\left.8.00^{\circ}\right)$

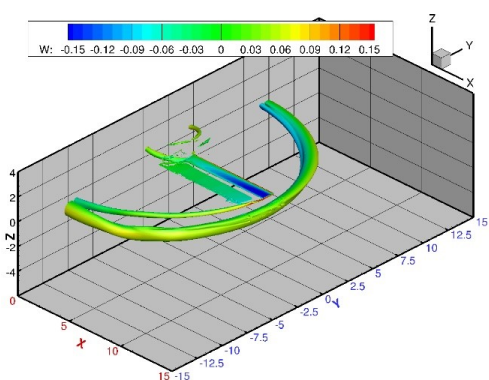

(b) $0.111 \mathrm{~s}$ (collective $4.44^{\circ}$ )

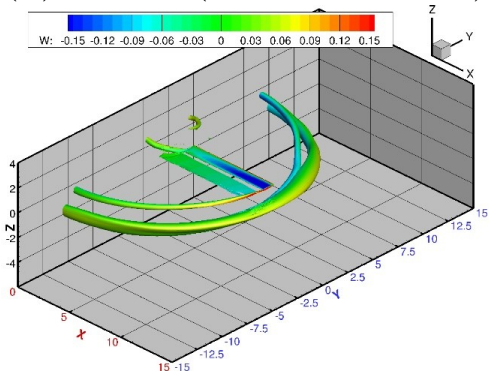

(e) $0.144 \mathrm{~s}$ (collective $5.78^{\circ}$ )

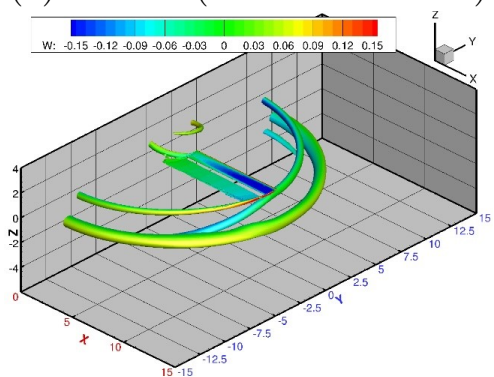

(h) $0.178 \mathrm{~s}\left(\right.$ collective $7.11^{\circ}$ )

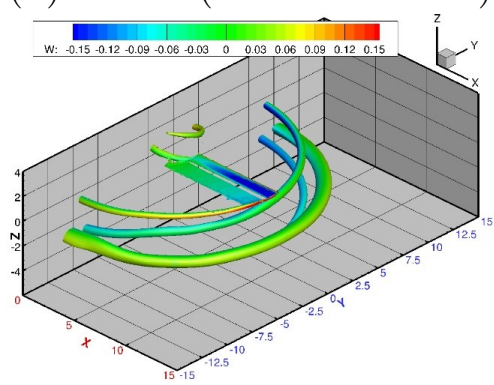

(k) $0.211 \mathrm{~s}\left(\right.$ collective $8.00^{\circ}$ )

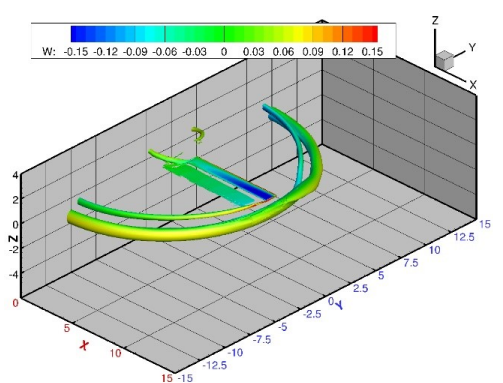

(c) $0.122 \mathrm{~s}\left(\right.$ collective $4.89^{\circ}$ )

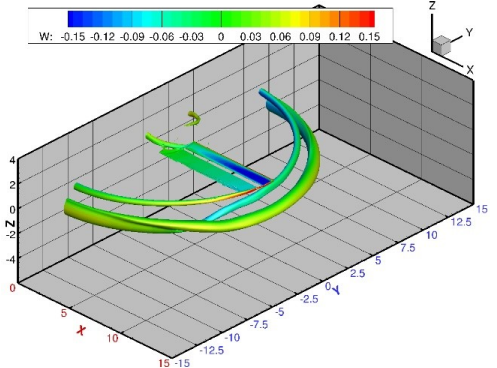

(f) $0.156 \mathrm{~s}$ (collective $6.22^{\circ}$ )

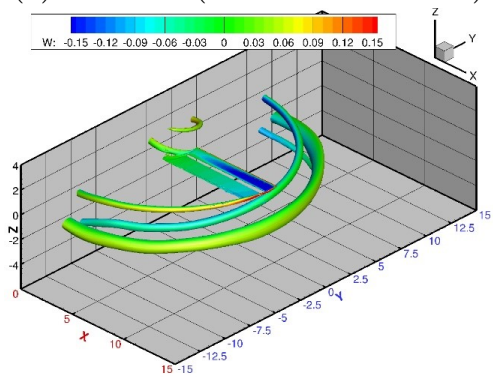

(i) $0.189 \mathrm{~s}\left(\right.$ collective $\left.7.56^{\circ}\right)$

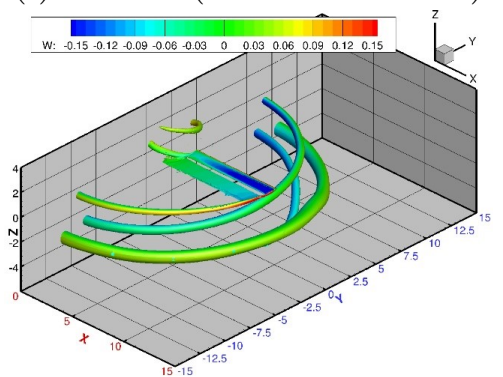

(l) $0.222 \mathrm{~s}$ (collective $8.00^{\circ}$ )

Figure 14: The wake geometry from CFD simulation with initial collective of 4 degrees. 
figure shows a rapidly changing wake that contains the starting vortex of the rotor and the new tip vortex that is changing position and strength due to the variable collective. The thrust of the rotor is rather low and for this reason the wake stays close to the blade and interacts strongly with it. At 0.133 s the strong initial vortex attracts the tip vortex and since they are co-rotating they wrap around each other. This continues up to $0.155 \mathrm{~s}$ where the blade begins to produce significant thrust $\mathrm{CT}>0.005$. At around 0.2 seconds the rotor produces enough thrust to push the initial vortex further below and the results suggest that a rake resembling closer to a rotor in steady hover is reached.

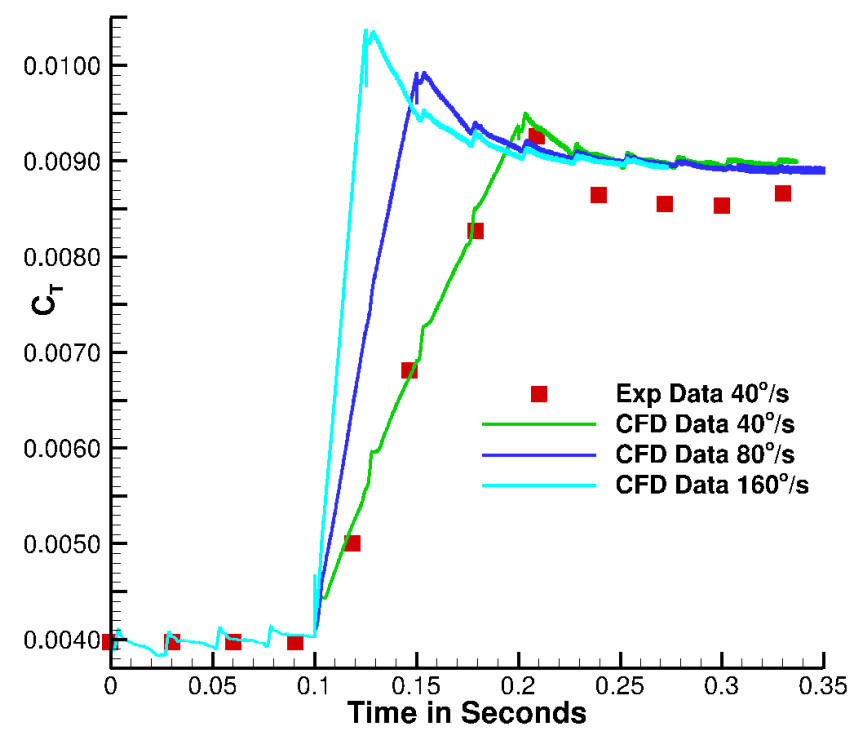

Figure 15: Comparison of the thrust of the rotor for the NUAA test with initial collective of 4 degrees for different collective pitch rates.

Figure 15 shows the effect of different collective pitch rates on the thrust of the rotor. As the rate is increased the transient overshoot also increases before decreasing to the long-term value between 2 and 3 turns after the collective has reached its final position. For the fastest collective pitch rate the overshoot is approximately 20\%. At the faster rates the velocity delta between pitching and non-pitching parts is larger and this results in a more pronounced change in thrust at the end of the pitch up cycle. However, the thrust recovers in the next couple of time steps demonstrating that the idealized abrupt change in velocity does not affect the overall thrust curve.

The vortex core position is also compared on a plane fixed at $20^{\circ}$ azimuth behind 


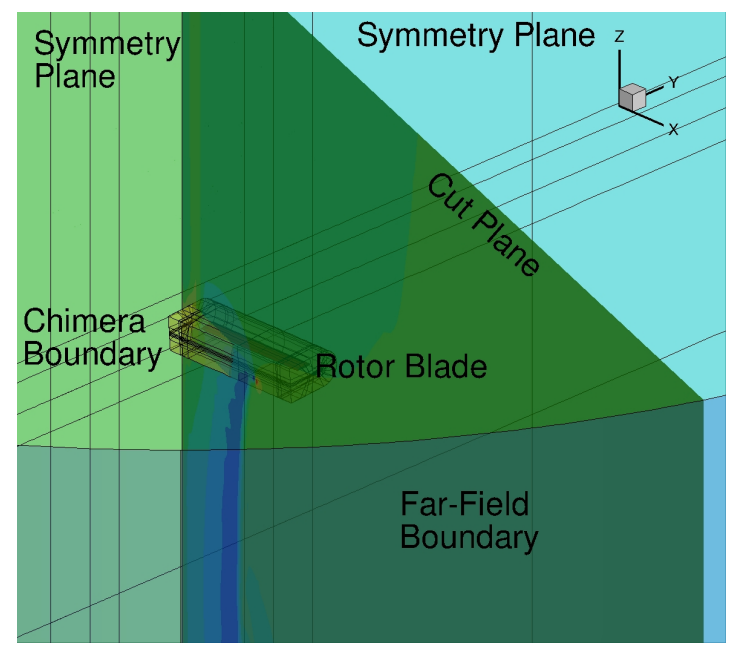

Figure 16: Schematic of the cut plane 20 degrees azimuth behind the rotor

the blade for both the free wake and CFD simulations. The position of this plane can be seen in the schematic 16 . There are many different vortex detection methods which can be split into two cases, firstly methods for finding vortex regions and secondly methods for finding vortex cores. Overviews of the different methods for vortex detection can be found in Roth [38] and Post et al. [39].

The initial approach was to assign the vortex core to the nearest grid point. The method for choosing the grid point was by finding the local maxima of Q-criterion $[40,41]$ on the fixed plane. The vertical position of the free wake core was shifted up 0.008934 and outboard 0.0012 of the rotor radii. This was done to account for the effect of the blade rollup over the blade which is not accounted for in the free wake model. The values were chosen so that the vertical positions co inside for the first vortex. This displacement is very small, $0.6 \mathrm{~mm}$ outboard and $4.5 \mathrm{~mm}$ upstream.

The use of the nearest grid point results in vortex core position moving from position to another in steps of the size of the cells. The cell size are approximately $3 \%$ percent of the blade chord $(1.6 \mathrm{~mm})$ and this determines the resolution in the vortex core position. This can be improved by either calculation on finer grids with the corresponding increase in computational cost or using a higher order interpolation around the local maxima on the cutting plane.

The higher order interpolation was achieved in MATLAB using a second order surface interpolation, (polyfitn[42]), with the local maxima and the 8 points surrounding it. The model was then differentiated symbolically for two orthogonal directions within the plane. The two equations are then solved for the final position of the local maxima. Figure 17 shows the comparison between the two methods 
as well as a curve estimating the position of the vortex core from the nearest grid point data.

For the first vortex cores only moves a couple of cells but the use of higher order interpolation smooths the variation out. It also shows that there is a $4 / \mathrm{rev}$ oscillation of approximately $0.5 \mathrm{~mm}$ which much less than the mesh resolution. The estimated position of the vortex core removes the $4 /$ rev oscillation in the CFD simulation as well as clipping both the under and overshoots slightly.

For the initial vortex there is very little change in position as the collective is ramped from $4^{\circ}$ to $8^{\circ}$. Both the radial and vertical positions change by less than $1 \%$ of the rotor radius. Examining figure 17(a) a very small overshoot of the radial position can be picked out in the interpolated solution which is completely hidden in the nearest point data. The second vortex also shows a same $4 / \mathrm{rev}$ oscillation and a small overshoot is now visible in both the radial and vertical position of the vortex. There is good agreement between the radial position for the second vortex and both solutions show a small (0.5\%) displacement outboard just after the collective pitch up occurs.

After approximately $0.025 \mathrm{~s}$ into the collective pitch up the radial position starts moving inboard. This inboard motion continues until about 1 revolution passed the end of the collective pitch change. The vertical position of the second vortex shows a very similar trend between the two methods however there a large offset between the two results. It should be noted there is a half revolution delay before the CFD starts reacting to the change in collective pitch while the free wake more has no such time lag.

In the third vortex the magnitude of the $4 /$ rev oscillation has increased by $50 \%$ in the radial direction and $33 \%$ in the vertical direction. The radial direction shows that the cortex core moves outboard for $3 / 4$ of the pitch up cycle before moving inboard. The core moves inboard for a complete revolution after the pitch up motion has finished. The vertical direction also shows a lag in both the initial movement of the core downwards and its maximum displacement. However in this case the maximum displacement occurs approximately half a revolution after the pitch up motion has finished.

As can be seen from the results that the effect of the change in pitch has a minor the position of the tip vortex its strength does increase which has a strong effect on the outboard loading on the blade. At larger wake ages the change in pitch 


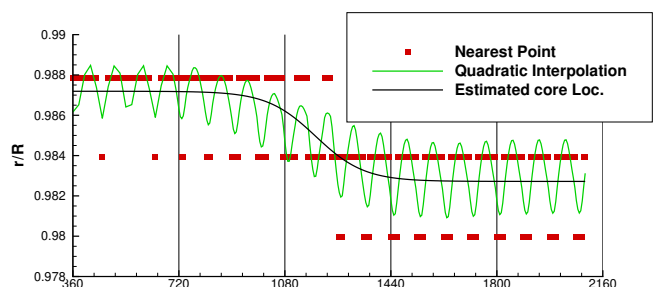

(a) Radial position of first vortex

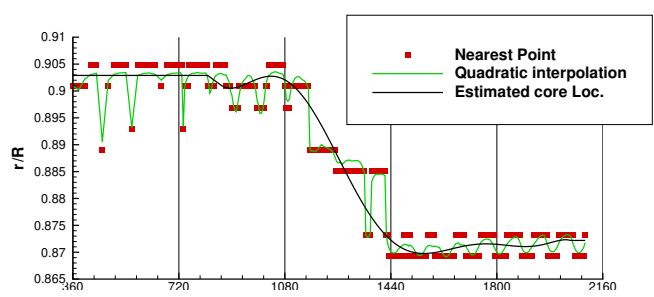

(c) Radial position of second vortex

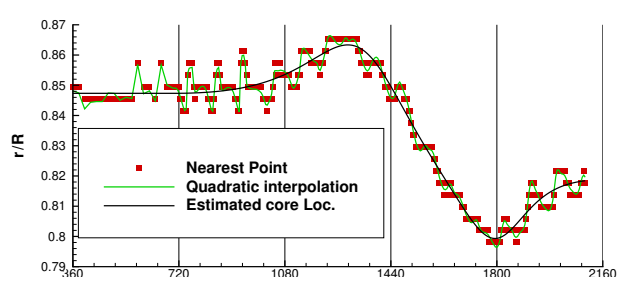

(e) Radial position of third vortex

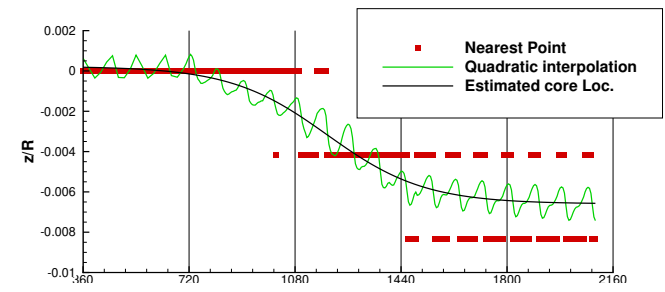

(b) Vertical position of first vortex

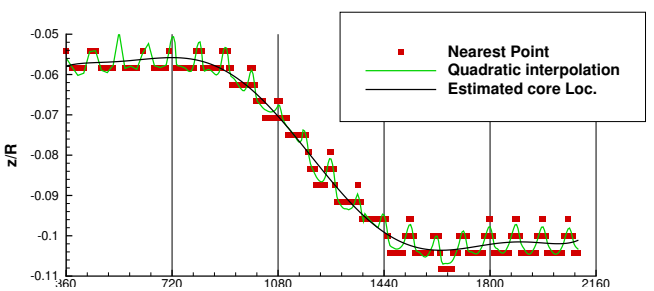

(d) Vertical position of second vortex

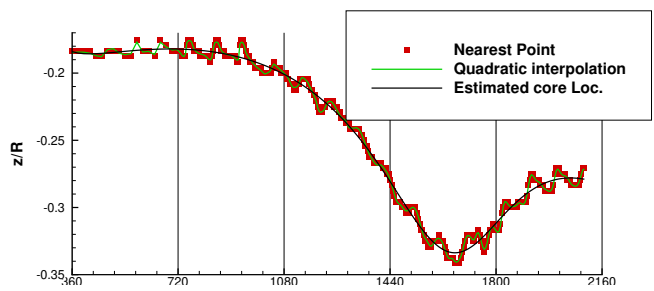

(f) Vertical position of third vortex

Figure 17: Comparison of vortex position $20^{\circ}$ behind the rotor blade for CFD simulations using two different vortex core detection methods with initial collective of 4 degrees.

has a much more marked effect on the position of the vortex and this also has a secondary effect of also changing the outboard loading. Due to this reason it is not easy to capture this effect using two dimensional strip theory due to the inherent three dimensionality of the flow.

Figure 18 shows the comparison of the estimated core location from the wake targeted background grid. As before the free wake solution for the vertical displacement has been matched with the CFD for the first vortex core. For the radial position there is an offset between the CFD and free wake positions but the tends are well represented. After the step input in collective pitch is complete the CFD solution converges to the new steady position faster. For the vertical core position the downwash is not advecting the CFD away from the blade as fast as the free wake solution. This results in an increasing offset between the two solutions. And while the trend during step input is well represented the CFD solution recovers to the converged position much quicker than the free wake solution.

Figure 19 shows the position of the vortex cores on a plane $20^{\circ}$ behind the rotor blade as different times. The position of the first core is nearly independent of the collective of the blade. The second core has just moved vertically downwards 


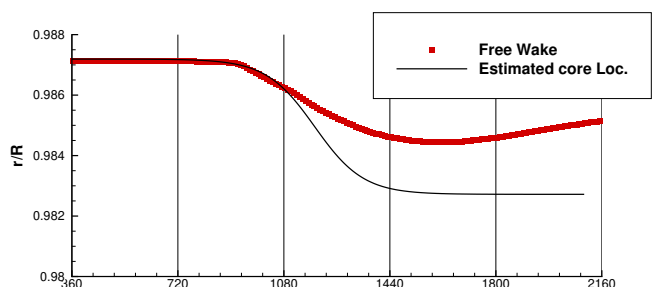

(a) Radial position of first vortex

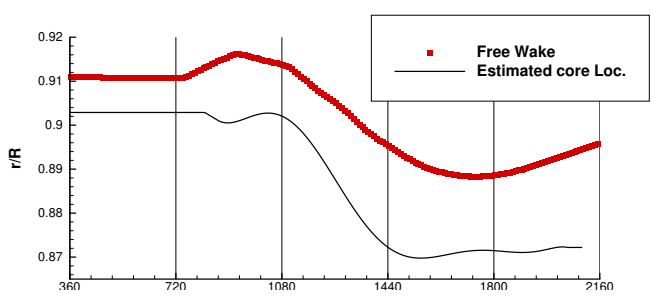

(c) Radial position of second vortex

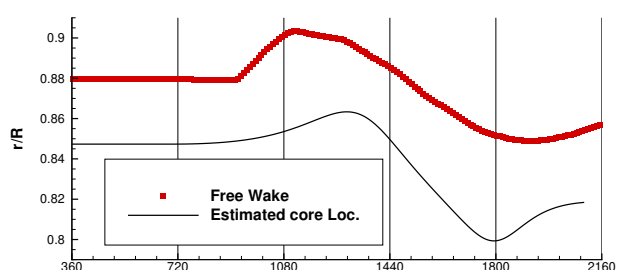

(e) Radial position of third vortex

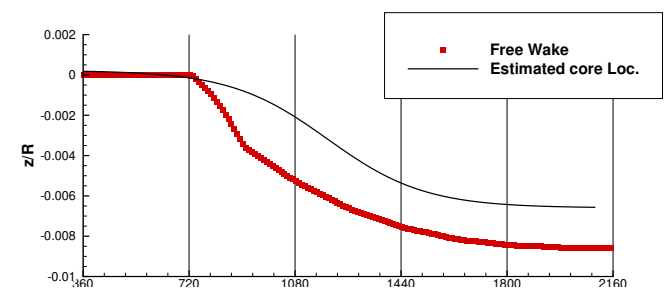

(b) Vertical position of first vortex

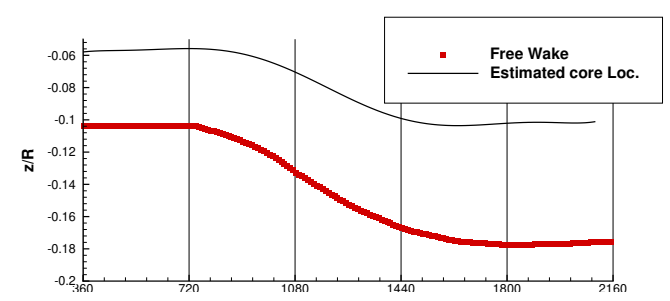

(d) Vertical position of second vortex

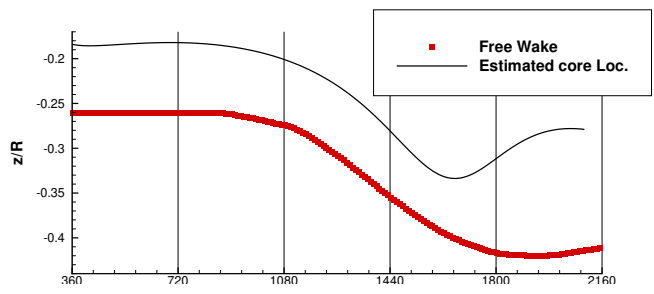

(f) Vertical position of third vortex

Figure 18: Comparison of vortex position $20^{\circ}$ behind the rotor blade for estimated core location from CFD and the free wake method with initial collective of 4 degrees.

halfway through the collective pitch change. After the collective pitch has reached it maximum the core moved both inboard and farther away from the rotor blade. The third core shows no vertical movement through the first half of collective pitch change, but the radial position moves outboard. In the second half of the collective pitch change the core moved away from the rotor blade while reversing direction in the radial direction and finishing up inboard of the initial position. The core has overshot its final position at about time $\mathrm{t}=0.3 \mathrm{~s}$ taking another rotor revolution to return close to its final position.

\section{Conclusions}

This work is a first step towards validation of CFD methods for changing inputs to rotors. The experiments of NUAA provide a simple though challenging test case for collective inputs in hover. The results of the CFD are in fair agreement with the test data when refined grids and high temporal resolution was used. The vortex dynamics shows how sensitive the position of the wake is to the initial conditions and its effect on transient response. The vortex dynamics shows some lag between 


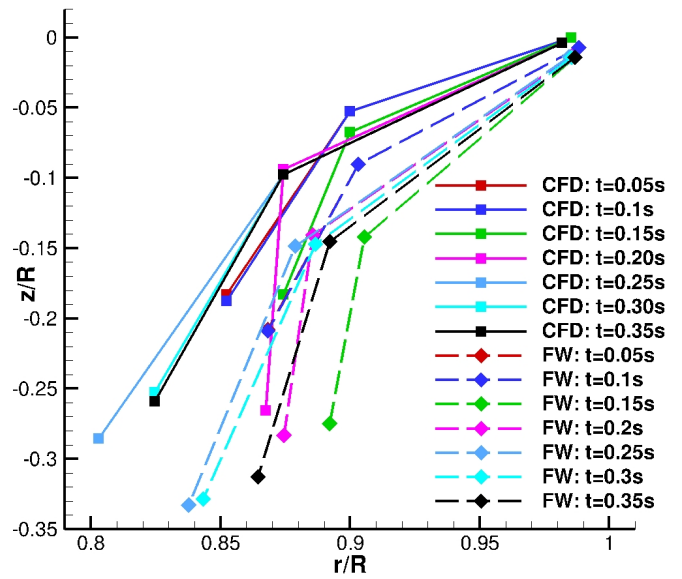

Figure 19: Position of the vortex cores on a plane $20^{\circ}$ behind the rotor blade.

the change in collective input and wake response. The vortex cores settle to an established wake after the collective input is finished but overshoot their positions as the wake adjusts to the changing rotor downwash.

The results show that only a single blade needs to be modelled in this case since the flow is periodic in space, reducing the computational cost of the calculation by the number of blades in the rotor. The overshoot in thrust at the maximum pitch rate of $160^{\circ}$ was about $17 \%$. The delta increase in thrust was proportionally smaller as the pitch rate increased.

This work clearly shows that CFD can provide the time evolution of the blade loads during the transient, however extracting the wake information is not a trivial task and further research is needed into vortex core identification on meshes which have grid resolution of $3-5$ percent of the rotor chord. The results do show however that it is possible to capture the transient behaviour of the wake using a relatively coarse background mesh of 20 million points. Even smaller background grids in the case of a higher number of blades.

Future work will look further into the wake dynamics and investigate inputs to other rotor control angles and vortex core identification within the CFD solve to increase azimuthal resolution with requiring complete flow fields to be stored.

\section{Acknowledgments}

The sponsorship of the MENtOR (EP/S013814/1) and National Natural Science Foundation of China (No. 11972190) is gratefully acknowledged. This work used the 
Cirrus UK National Tier-2 HPC Service at EPCC (http://www.cirrus.ac.uk) project ec004 and the ARCHER UK National Supercomputing Service (http://www.archer.ac.uk) project e613.

\section{References}

[1] Komerath, N. M., Smith, M. J., and Tung, C., "A review of rotor wake physics and modeling," Journal of the American Helicopter Society, Vol. 56, No. 2, 2011, pp. 22006.1-19.

[2] Van Hoydonck, W. R. M., Haverdings, H., and Pavel, M. D., "A review of rotorcraft wake modeling methods for flight dynamics applications," $35^{\text {th }}$ European Rotorcraft Forum, 2009.

[3] Drees, J. M., "A theory of airflow through rotors and its application to some helicopter problems," J. Helicopter Association of Great Britain, Vol. 3, No. 2, 1949, pp. 79-104.

[4] Landgrebe, A. J., "An analytical method for predicting rotor wake geometry," Journal of the American Helicopter Society, Vol. 14, No. 4, 1969, pp. 20-32.

[5] Bagai, A. and Leishman, J. G., "Rotor free-wake modeling using a pseudoimplicit technique - including comparisons with experimental data," Journal of the American Helicopter Society, Vol. 40, No. 3, 1995, pp. 29-41.

[6] Strawn, R. C. and Caradonna, F. X., "Conservative full-potential model for unsteady transonic rotor flows," AIAA Journal, Vol. 25, No. 2, 1987, pp. 193 198.

[7] Brown, R. E. and Line, A. J., "Efficient high-resolution wake modeling using the vorticity transport equation," AIAA Journal, Vol. 43, No. 7, 2005, pp. 14341443.

[8] He, C. and Zhao, J., "Modeling rotor wake dynamics with viscous vortex particle method," AIAA Journal, Vol. 47, No. 4, 2009, pp. 902-915.

[9] Tan, J. F. and Wang, H. W., "Simulating unsteady aerodynamics of helicopter rotor with panel/viscous vortex particle method," Aerospace Science and Technology, Vol. 30, No. 1, 2013, pp. 255-268. 
[10] Ahmad, J. and Duque, E. P., "Helicopter rotor blade computation in unsteady flows using moving overset grids," Journal of Aircraft, Vol. 33, No. 1, 1996, pp. 54-60.

[11] Shi, Y., Xu, G., and Wei, P., "Rotor wake and flow analysis using a coupled Eulerian-Lagrangian method," Engineering Applications of Computational Fluid Mechanics, Vol. 10, No. 1, 2016, pp. 384-402.

[12] Carpenter, P. J. and Fridovich, B., "Effect of a rapid blade-pitch increase on the thrust and induced-velocity response of a full-scale helicopter rotor," $N A C A$ $T N-3044,1953$.

[13] Ellenrieder, T. and Brinson, P., "The dynamic induced velocity field of a model rotor in hover conditions," The Aeronautical Journal, Vol. 102, No. 1016, 1998, pp. 331-336.

[14] Jessurun, K., Pavel, M., and Toet, S., "Apparent mass effects on a stiff-hinged rotor model after rapid cyclic and/or collective inputs - a flow visualization study," 27th European Rotorcraft Forum, Moscow, Russia, 2001.

[15] Pitt, D. M. and Peters, D. A., "Theoretical prediction of dynamic - inflow derivatives," Vertica, Vol. 5, 1981, pp. 21-34.

[16] Peters, D. A. and He, C. J., "Finite state induced flow models. II - Threedimensional rotor disk," Journal of Aircraft, Vol. 32, No. 2, 1995, pp. 323-333.

[17] Sadler, S. G., "Main rotor free wake geometry effects on blade air loads and response for helicopters in steady maneuvers. Volume 1: Theoretical formulation and analysis of results," NASA CR-2110, 1972.

[18] Bhagwat, M. J. and Leishman, J. G., "Rotor aerodynamics during maneuvering flight using a time-accurate free-vortex wake," Journal of the American Helicopter Society, Vol. 48, No. 3, 2003, pp. 143-158.

[19] Bhagwat, M. J. and Leishman, J. G., "Time-accurate free-vortex wake model for dynamic rotor response," American Helicopter Society Specialist meeting, Atlanta, 2000.

[20] Duraisamy, K. and Brown, R., "Aerodynamic response of a hovering rotor to ramp change in pitch input," $64^{\text {th }}$ American Helicopter Society Annual Forum, 2008. 
[21] Strawn, R. C., Caradonna, F. X., and Duque, E. P., "30 years of rotorcraft computational fluid dynamics research and development," Journal of the American Helicopter Society, Vol. 51, No. 1, 2006, pp. 5-21.

[22] Bhagwat, M. J., Moulton, M. A., and Caradonna, F. X., "Development of a CFD-based hover performance prediction tool for engineering analysis," Journal of the American Helicopter Society, Vol. 52, No. 3, 2007, pp. 175-188.

[23] Barakos, G. and Garcia, A. J., "CFD analysis of hover performance of rotors at full-and model-scale conditions," The Aeronautical Journal, Vol. 120, No. 1231, 2016, pp. 1386-1424.

[24] Duraisamy, K. and Baeder, J. D., "High resolution wake capturing methodology for hovering rotors," Journal of the American Helicopter Society, Vol. 52, No. 2, 2007, pp. 110-122.

[25] Rajmohan, N., Manivannan, V., Sankar, L., Costello, M., and Bauchau, O., "Development of a methodology for coupling rotorcraft aeromechanics and vehicle dynamics to study helicopters in maneuvering flight," $65^{\text {th }}$ American Helicopter Society Annual Forum, 2009.

[26] Rajmohan, N., Application of hybrid methodology to rotors in steady and maneuvering flight, Ph.D. thesis, Georgia Institute of Technology, 2010.

[27] Thomas, S., Ananthan, S., and Baeder, J. D., "Wake-coupling CFD-CSD analysis of helicopter rotors in steady and maneuvering flight conditions," American Helicopter Society Specialist's Conference on Aeromechanics, 2010.

[28] Bhagwat, M. J., Ormiston, R. A., Saberi, H. A., and Xin, H., "Application of computational fluid dynamics/computational structural dynamics coupling for analysis of rotorcraft airloads and blade loads in maneuvering flight," Journal of the American Helicopter Society, Vol. 57, No. 3, 2012, pp. 1-21.

[29] Li, P. and Chen, R., "Rotor unsteady aerodynamics model using an efficient free-vortex method," Aircraft Engineering and Aerospace Technology, 2012.

[30] Steijl, R., Barakos, G., and Badcock, K., "A framework for CFD analysis of helicopter rotors in hover and forward flight," International Journal for Numerical Methods in Fluids, Vol. 51, No. 8, 2006, pp. 819-847.

[31] Jameson, A., "Time Dependent Calculations Using Multigrid, with Applications to Unsteady Flows Past Airfoils and Wings," $10^{\text {th }}$ Computational Fluid Dynamics Conference, Honolulu, HI, 24-26 June 1991, AIAA-1991-1596. 
[32] Osher, S. and Chakravarthy, S., "Upwind schemes and boundary conditions with applications to Euler equations in general geometries," Journal of Computational Physics, Vol. 50, No. 3, 1983, pp. 447-481.

[33] Van Albada, G. D., Van Leer, B., and Roberts, W. W., "A comparative study of computational methods in cosmic gas dynamics," Upwind and high-resolution schemes, Springer, 1997, pp. 95-103.

[34] Menter, F. R., "Two-equation eddy-viscosity turbulence models for engineering applications," AIAA Journal, Vol. 32, No. 8, 1994, pp. 1598-1605.

[35] Li, P., "Rotor unsteady free-vortex wake model and investigation on highfidelity modeling of helicopter flight dynamics," Nanjing University of Aeronautics and Astronautics, Nanjing, 2010.

[36] Peters, D. A., "How dynamic inflow survives in the competitive world of rotorcraft aerodynamics," Journal of the American Helicopter Society, Vol. 54, No. 1, 2009, pp. 11001-11001.

[37] Pitt, D. M. and Peters, D. A., "Theoretical prediction of dynamic-inflow derivatives," Vertica, Vol. 5, No. 1, 1981, pp. 21-34.

[38] Roth, M., Automatic extraction of vortex core lines and other line-type features for scientific visualization, Ph.D. thesis, Swiss Federal Institute of Technology, ETH Zurich, 2000, Diss. ETH No. 13673.

[39] Post, F. H., Vrolijk, B., Hauser, H., Laramee, R. S., and Doleisch, H., "The State of the Art in Flow Visualisation: Feature Extraction and Tracking," Computer Graphics Forum, Vol. 22, No. 4, 2003, pp. 775-792.

[40] Hunt, J. C., Wray, A. A., and Moin, P., "Eddies, streams, and convergence zones in turbulent flows," Center for Turbulence Research Report CTR-S88, 1988, pp. $193-208$.

[41] Koláŕ, V., "Vortex identification: New requirements and limitations," International Journal of Heat and Fluid Flow, Vol. 28, No. 4, 2007, pp. 638 - 652, Including Special Issue of Conference on Modelling Fluid Flow (CMFF'06), Budapest.

[42] D'Errico, J., "Polynomial modeling in 1 or n dimensions," http://https:// uk.mathworks.com/matlabcentral/fileexchange/34765-polyfitn, 2006, Accessed: 13-08-2020. 


\section{A MATLAB code of the dynamic inflow model}

Listing 1: Dynamic inflow model

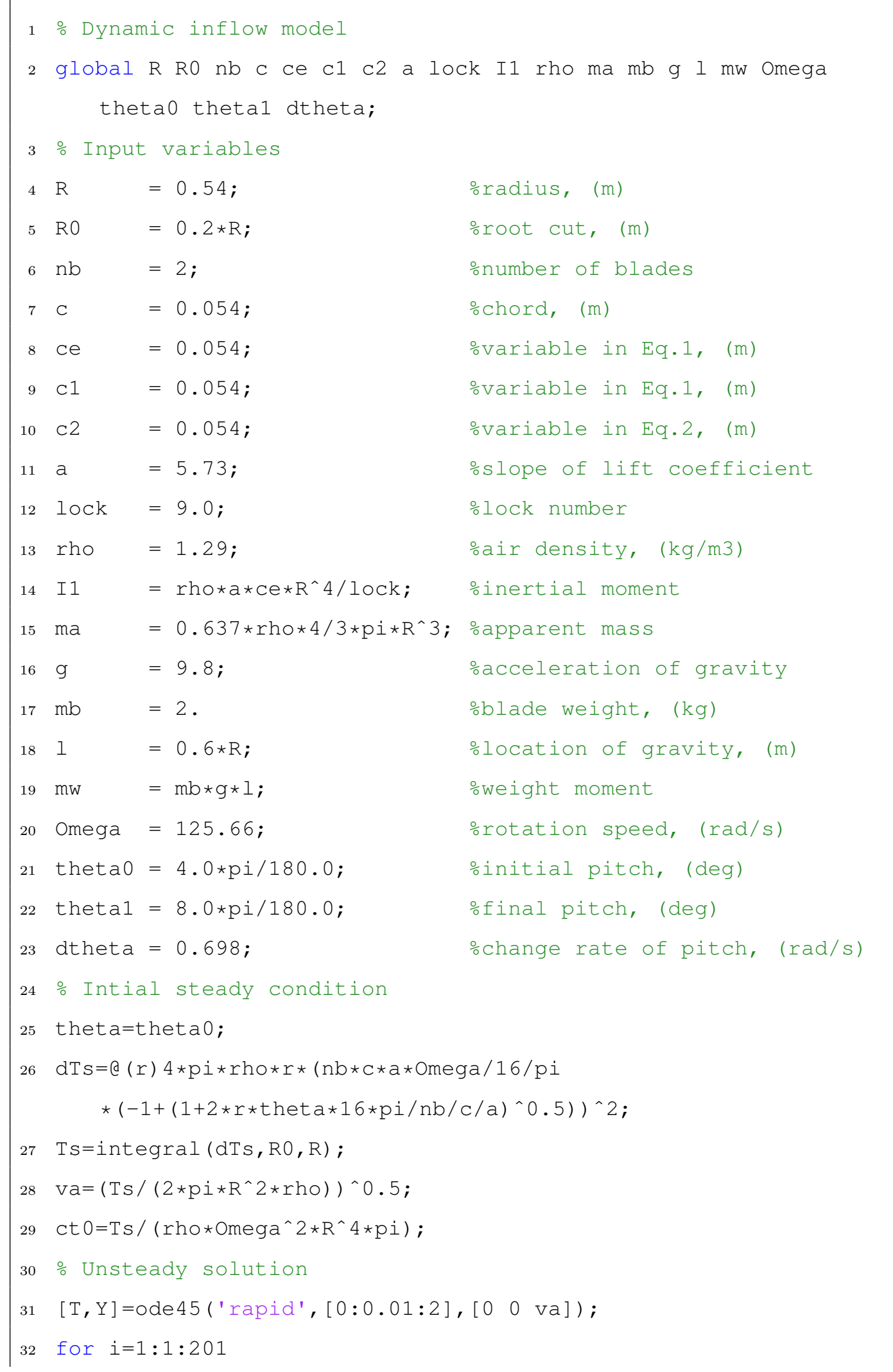




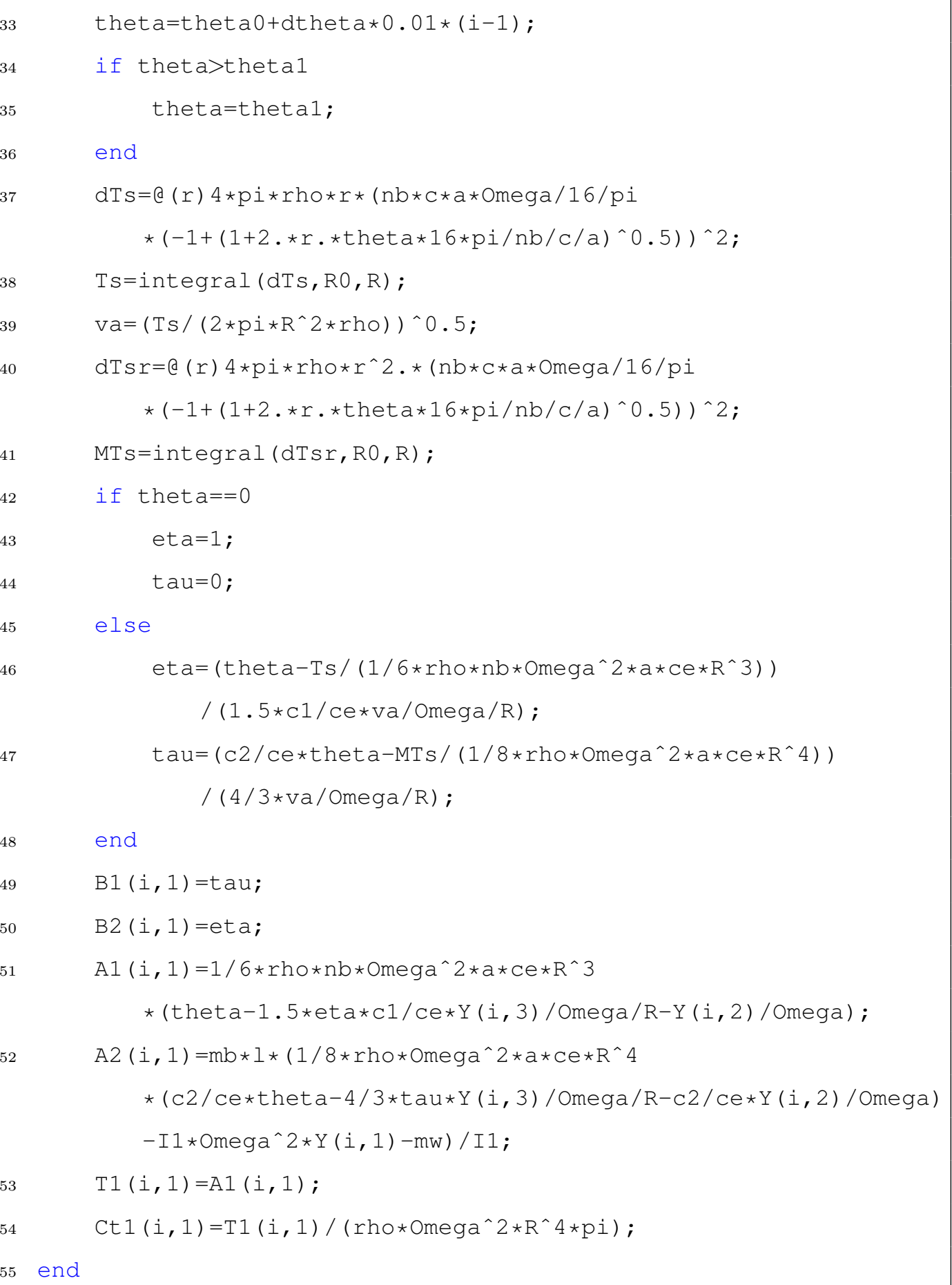

Listing 2: Function rapid

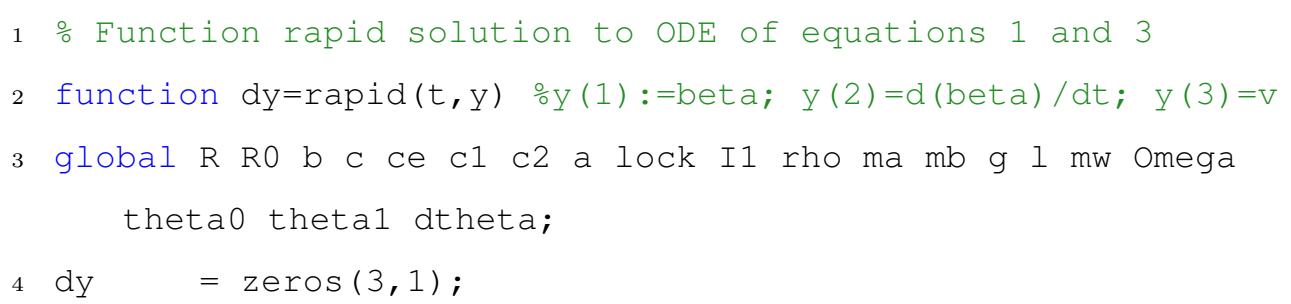




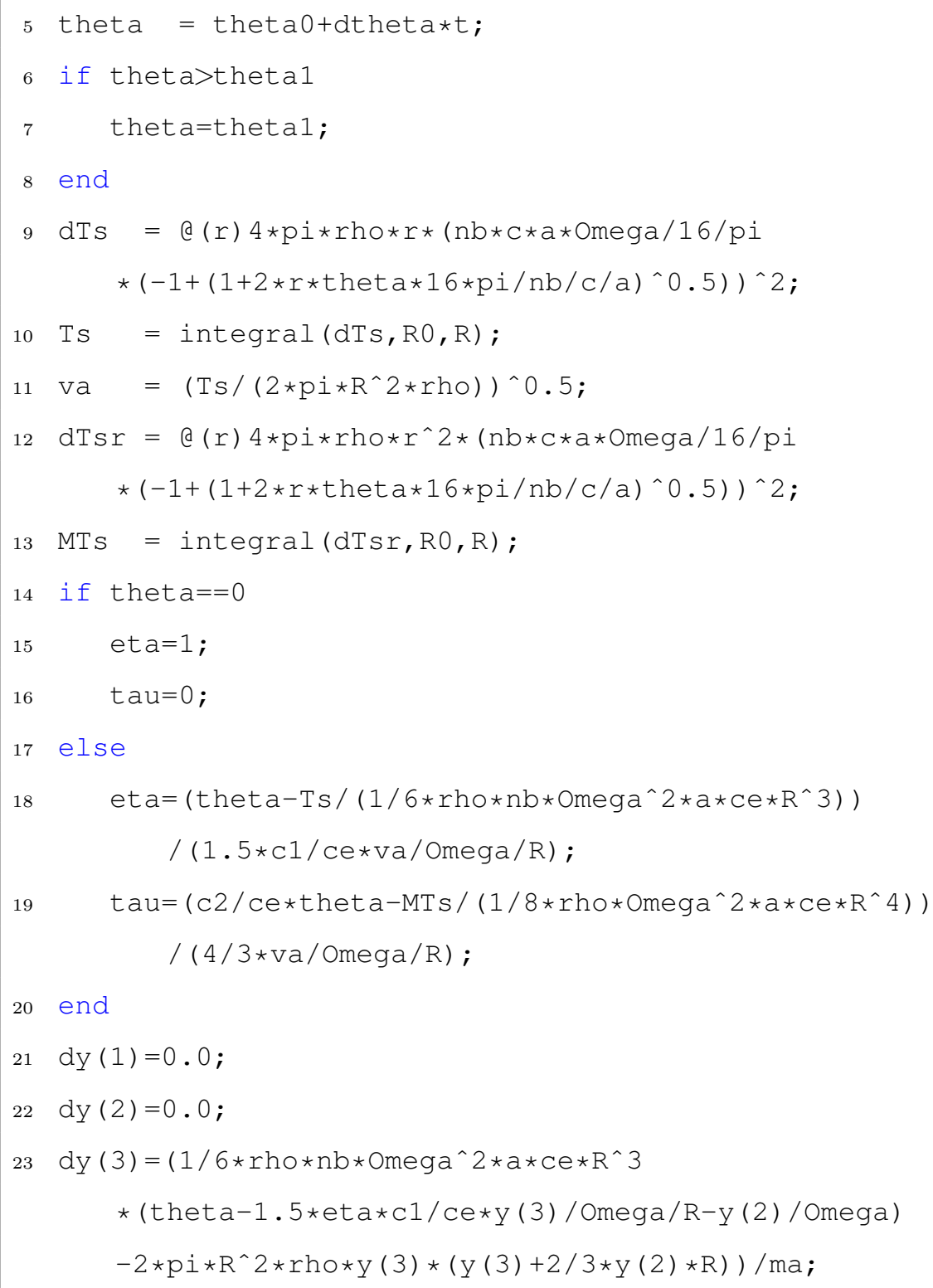

